\title{
Influence of Saliva and Mucin on the Adhesion of Candida Oral Clinical Isolates
}

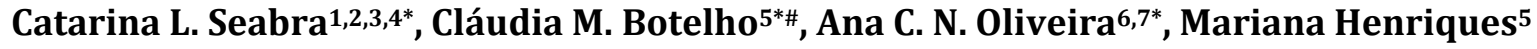 \\ ${ }^{1}$ Instituto de investigação e Inovação em Saúde, University of Porto, Porto, Portugal \\ ${ }^{2}$ Instituto de Engenharia Biomédica, University of Porto, Porto, Portugal \\ ${ }^{3}$ Instituto de Patologia e Imunologia Molecular da Universidade do Porto, University of Porto, Porto, Portugal \\ ${ }^{4}$ Instituto de Ciências Biomédicas Abel Salazar, University of Porto, Porto, Portugal \\ ${ }^{5}$ Centre of Biological Engineering (CEB), Laboratório de Investigação em Biofilmes Rosário Oliveira, \\ University of Minho, Braga, Portugal \\ ${ }^{6}$ Centre of Physics (CFUM), Department of Physics, University of Minho, Braga, Portugal \\ ${ }^{7}$ Centre of Molecular and Environmental Biology (CBMA), Department of Biology, University of Minho, Braga, \\ Portugal \\ Email: "claudiabotelho@deb.uminho.pt
}

Received 12 March 2015; accepted 27 December 2015; published 30 December 2015

Copyright $(2015$ by authors and Scientific Research Publishing Inc.

This work is licensed under the Creative Commons Attribution International License (CC BY).

http://creativecommons.org/licenses/by/4.0/

(C) () Open Access

\begin{abstract}
Objectives: This research work intends to clarify the role of artificial saliva, in particularly the role of mucin, a salivary protein, on the surface properties and adhesion ability of Candida spp. oral clinical isolates to abiotic surfaces. Methods: Four oral clinical isolates of Candida spp. were used: two Candida albicans strains (AC; AM) and two Candida parapsilosis strains (AD; AM2). The strains were isolated from patients using oral prosthesis. The microorganisms were cultured in the absence or presence of mucin and artificial saliva, and their adhesion to an abiotic surface (coated with mucin and artificial saliva) was evaluated. Results: The presence of mucin per se onto the abiotic surface decreased the adhesion of all strains, although the combination of mucin with artificial saliva had reduced this effect. No direct correlation between adhesion and the surface free energies of adhesion of the microorganisms was found. Significance: Candida spp. were human commensal microorganisms that became pathogenic when the host immune defenses were compromised. Medical devices were colonized by Candida spp. particularly, oral prostheses, which might lead to the degradation of the prostheses and systemic infections. The salivary secretions that constantly cover the oral cavity influenced Candida spp. adhesion process. Therefore, it was important to understand the interactions between Candida spp., salivary proteins and the characteristic of oral prosthesis when developing materials for oral prostheses.
\end{abstract}

${ }^{*}$ Equal contribution.

${ }^{\#}$ Corresponding authors.

How to cite this paper: Seabra, C.L., Botelho, C.M., Oliveira, A.C.N. and Henriques, M. (2015) Influence of Saliva and Mucin on the Adhesion of Candida Oral Clinical Isolates. Journal of Encapsulation and Adsorption Sciences, 5, $217-227$.

http://dx.doi.org/10.4236/jeas.2015.54018 
Keywords

Candida, Artificial Saliva, Mucin, Oral Adhesion, Surface Properties

\section{Introduction}

Candida spp. are opportunistic microorganisms present in the normal microbiota. On the right environment, these microorganisms are able to colonize, invade and multiply in tissues and organs, causing fungal infections that can go from superficial lesions to systemic infections [1] [2]. The ability of Candida spp. to adhere to host cells and inert substrates is one of the main driving forces for its pathogenicity, often leading to colonization, infection, and formation of biofilms [3].

Candida spp. are found in the oral cavity of more than $50 \%$ of the human population, and $80 \%$ of the clinical isolates are identified as Candida albicans. Candida parapsilosis is an emerging human pathogen that has dramatically increased in significance and prevalence over the past 2 decades, such that $C$. parapsilosis is now one of the leading causes of invasive candidal disease [4].

The human organism has many defence mechanisms in order to avoid colonization by microorganisms. Mucosal epithelial cells continuously secrete a mucosal fluid that acts as a barrier to maintain a healthy mucosa. In addition, saliva is formed by many defensive compounds, including mucins, antibodies, lysozyme or histatins that regulate the microorganism populations in the oral cavity [5]. Mucins are a major component of saliva. These large glycoproteins, with a high degree of glycosylation and potential for hydration, present antimicrobial activity, and opsonization ability, and are important components of the acquired pellicle [5] [6]. Nevertheless, saliva is also a source of water, nutrients and adherence factors. Salivary proteins can adsorb onto oral surfaces like tooth enamel and dentures, forming the acquired enamel pellicle [7]-[9], to which microorganisms are then able to adhere. Therefore, the precise role of saliva on Candida spp. adhesion to dentures is controversial. Several studies have shown that saliva reduces the adherence of $C$. albicans to dentures and epithelial cells [9], but other authors describe that saliva enhances the adherence of Candida to polystyrene [10] and polymethylmethacrylate [11].

Salivary secretions constantly cover the oral cavity, so, it is important that, during the investigation of oral colonization, the interactions between Candida spp. and salivary proteins are considered. This research work intends to clarify the role of the salivary protein mucin, as well as artificial saliva, on the surface properties and the adhesion ability of Candida spp. oral clinical isolates.

\section{Materials and Methods}

\subsection{Microbial Strains}

Four oral clinical isolates of Candida spp. were used in this study: two isolates of Candida albicans (strains AC and AM) and two isolates of Candida parapsilosis (strains AD and AM2). The strains were isolated from patients using an oral prosthesis. Candida albicans AC and Candida parapsilosis AD were obtained from different individuals and Candida albicans AM and Candida parapsilosis AM2 from the same individual. The oral isolates were obtained from a Dentistry Clinic and belong to the Biofilm Group of the Centre of Biological Engineering, Minho University, where they were identified by molecular methods.

\subsection{Culture Conditions and Conditioning Mediums}

Candida isolates were subcultured on Sabouraud dextrose agar for $24 \mathrm{~h}$ at $37^{\circ} \mathrm{C}$, after which each strain was inoculated in Sabouraud dextrose broth for $16 \mathrm{hrs}$ at $37^{\circ} \mathrm{C}$ in an orbital shaker at $120 \mathrm{rpm}$. Cells were then harvested by centrifugation at $8000 \mathrm{rpm}$ and washed twice with phosphate saline buffer (PBS, pH 7, $0.1 \mathrm{M}$ ). The cell pellets were resuspended in the conditioning mediums, and the cell concentration adjusted to $1 \times 10^{7}$ cells ml ${ }^{-1}$.

Three different media were used in this study, namely artificial saliva without mucin (AS-Mu) and with mucin (AS + Mu) and mucin in PBS (Mu). The artificial saliva was prepared according to Lamfon et al. [12]: 2 $\mathrm{gl}^{-1}$ yeast extract (Liofilchem, Italy), $5 \mathrm{gl}^{-1}$ peptone (Liofilchem, Italy), $2 \mathrm{gl}^{-1}$ glucose (Applichem, Germany), 1 $\mathrm{gl}^{-1}$ mucin from bovine submaxillary glands-Type I-S (Sigma-Aldrich, USA), $0.35 \mathrm{gl}^{-1} \mathrm{NaCl}$ (Applichem, 
Germany), $0.2 \mathrm{gl}^{-1} \mathrm{CaCl}_{2}$ (Riedel-de-Ha“en, Germany), and $0.2 \mathrm{gl}^{-1} \mathrm{KCl}$ (Pronalab, Portugal) (pH 6.8 - 7).

\subsection{Surface Coating with the Conditioning Media}

A 6-well polystyrene plate was used as an abiotic substrate to study the influence of the presence of mucin on the adhesion properties of Candida spp. The three different conditioning media were used for coating. The polystyrene surfaces were incubated with each conditioning medium for $4 \mathrm{hrs}$ at $37^{\circ} \mathrm{C}$ in an orbital shaker at $120 \mathrm{rpm}$, as described by Guggenheim et al. [13]. After the 4h incubation period, the wells were washed and stored in PBS at $4^{\circ} \mathrm{C}$ until needed (for a maximum of one week).

\subsection{Contact Angle Measurement and Determination of Surface Free Energies}

Contact angles were measured by the sessile drop technique, using a contact angle measurement apparatus model OCA 15 PLUS, DATAPHYSICS. Measurements were performed at room temperature using three different standard liquids (ultrapure water, formamide, and $\alpha$-bromonaphthalene). Every assay was performed in triplicate and at least 10 measurements were performed for each sample.

Contact angles were measured on yeast lawns. Briefly, microorganisms were grown for $2 \mathrm{~h}$ in artificial saliva without mucin (AS - Mu) or in artificial saliva with mucin (AS + Mu). Subsequently, the cell suspensions were layered onto $0.22 \mu \mathrm{m}$ pore sized filters and dried for $4 \mathrm{~h}$ at $37^{\circ} \mathrm{C}$, to standardize the humidity level [14].

The hydrophobicity and surface tension was determined using the Van Oss approach [15]-[17]. The acid-base nature of the surfaces was directly determined in terms of the surface free energy components $\gamma_{s v}^{L W}$ (LW, Lifshitz-Van der Waals) and $\gamma_{s v}^{A B}$ (AB, acid-base), according to Equation (1).

$$
\gamma_{s v}=\gamma_{s v}^{L W}+\gamma_{s v}^{A B}
$$

in which the $\mathrm{AB}$ component equals

$$
\gamma_{s v}^{A B}=2 \sqrt{\gamma_{s v}^{-} \times \gamma_{s v}^{+}}
$$

where $\gamma_{s v}^{-}$and $\gamma_{s v}^{+}$are the electron-donating and electron-accepting surface free energy parameters, respectively. $s$ stands for solid, $v$ for vapor and $l$ for liquid. Proper diagnostic liquids with known surface free energy components $\left(\gamma_{l v}^{L W}, \gamma_{l v}^{A B}, \gamma_{l v}^{+}\right.$and $\left.\gamma_{l v}^{-}\right)$were selected.

Since $\alpha$-bromonaphthalene is apolar $\left(\gamma_{l_{v}}^{A B}=0\right)$, its contact angle on a surface can be used to calculate the $\gamma_{s v}^{L W}$ component of the surface free energy

$$
\gamma_{s v}^{L W}=\left(\left(\sqrt{\gamma_{l v}} \times(\cos \theta+1)\right) / 2\right)^{2}
$$

Water and formamide are both polar liquids and their contact angles can be used to calculate $\gamma_{s v}^{-}$and $\gamma_{s v}^{+}$ from the Young equation Equation (4).

$$
\gamma_{l v} \times(\cos \theta+1)-2 \sqrt{\gamma_{s v}^{L W} \gamma_{l v}^{L W}}=2 \sqrt{\gamma_{s v}^{-} \gamma_{l v}^{+}}+2 \sqrt{\gamma_{s v}^{+} \gamma_{l v}^{-}}
$$

The free energy of adhesion $\Delta G_{a d h}$ can be separated into two components: $\Delta G_{a d h}^{L W}$ is the free energy of adhesion due to Lifshitz-van der Waals interactions, and $\Delta G_{a d h}^{A B}$ the free energy of adhesion due to electrostatic interactions, according to Equation (5).

$$
\Delta G_{a d h}=\Delta G_{a d h}^{L W}+\Delta G_{a d h}^{A B}
$$

where

$$
\Delta G_{a d h}^{L W}=-2 \times\left(\sqrt{\gamma_{m v}^{L W}}-\sqrt{\gamma_{l v}^{L W}}\right) \times\left(\sqrt{\gamma_{s v}^{L W}}-\sqrt{\gamma_{l v}^{L W}}\right)
$$

and

$$
\begin{aligned}
\Delta G_{a d h}^{A B}= & 2\left(\sqrt{\gamma_{m v}^{+}}-\sqrt{\gamma_{s v}^{+}}\right)\left(\sqrt{\gamma_{m v}^{-}}-\sqrt{\gamma_{s v}^{-}}\right)-2\left(\sqrt{\gamma_{m v}^{+}}-\sqrt{\gamma_{l v}^{+}}\right)\left(\sqrt{\gamma_{m v}^{-}}-\sqrt{\gamma_{l v}^{-}}\right) \\
& -2\left(\sqrt{\gamma_{s v}^{+}}-\sqrt{\gamma_{l v}^{+}}\right)\left(\sqrt{\gamma_{s v}^{-}}-\sqrt{\gamma_{l v}^{-}}\right)
\end{aligned}
$$




\subsection{Yeast Adhesion Assays}

The adhesion assays were performed on non-treated polystyrene surfaces, and polystyrene surfaces treated with the three different conditioning mediums: AS - Mu, AS + Mu and Mu. Yeast suspensions grown in AS - Mu or AS + Mu were used. Briefly, $500 \mu \mathrm{l}$ of the standardized cell suspensions $\left(1 \times 10^{7}\right.$ cells ml ${ }^{-1}$ in AS $-\mathrm{Mu}$ or AS + $\mathrm{Mu}$ ) were placed on the coated or uncoated well-plates for 2 hrs at $37^{\circ} \mathrm{C}$ in an orbital shaker at $120 \mathrm{rpm}$. Following this incubation period, the medium was removed and the wells washed with PBS to remove unattached cells. The wells were scraped to resuspend the adhered cells in PBS. The yeast cells were then sonicated for 45 sec, 30 W, in an Ultrasonic Processor. Viable counts for each Candida spp. were obtained by serial decimal dilutions in PBS, and plated on Sabouraud dextrose agar medium, followed by an incubation period of $24 \mathrm{hrs}$ at $37^{\circ} \mathrm{C}$.

\subsection{Statistical Analysis}

Statistical analysis was performed using the SPSS software. The one-way ANOVA test followed by a Bonferroni as a Post Hoc test (confidence level of 95\%) was applied.

\section{Results}

\subsection{Effect of Conditioning Medium on Polystyrene Surface Characteristics}

The treatments with AS - Mu, AS + Mu and Mu strongly affected the characteristics of the surface. The contact angles measured with the polar liquids (water and formamide) on the treated surfaces were lower than on the control surfaces (untreated), while the apolar liquid ( $\alpha$-bromonaftalene) formed higher contact angles (Table 1).

The surface free energy (total surface tension, composed by the Lifshitz-van der Walls $\gamma^{L W}$ and acid-base $\gamma^{A B}$ components) and hydrophobicity of treated and non-treated polystyrene surfaces was determined (Figure 1).

Table 1. Averaged contact angle values measured on the non-treated and treated polystyrene surfaces. Three standard liquids were used: water, formamide, and $\alpha$-bromonaftalene. Average \pm standard error (SE) are represented. NT-non-treated polystyrene surface; AS - Mu-polystyrene surface treated with artificial saliva without mucin; AS + Mu-polystyrene surface treated with artificial saliva with mucin; Mu-polystyrene surface treated with mucin in PBS.

\begin{tabular}{cccc}
\hline & $\boldsymbol{\theta}_{\text {water }}\left({ }^{\circ}\right) \pm \mathbf{S E}$ & $\boldsymbol{\theta}_{\text {formanide }}\left({ }^{\circ}\right) \pm \mathbf{S E}$ & $\boldsymbol{\theta}_{\boldsymbol{\alpha} \text {-bromonaftalene }}\left({ }^{\circ}\right) \pm \mathbf{S E}$ \\
\hline $\mathbf{N T}$ & $66.72 \pm 2.08$ & $49.84 \pm 1.95$ & $<0$ \\
$\mathbf{A S}-\mathbf{M u}$ & $<0$ & $29.27 \pm 1.46$ & $28.17 \pm 2.25$ \\
$\mathbf{A S}+\mathbf{M u}$ & $<0$ & $34.66 \pm 1.71$ & $39.99 \pm 1.34$ \\
$\mathbf{M u}$ & $28.76 \pm 1.52$ & $35.65 \pm 1.37$ & $41.75 \pm 3.32$ \\
\hline
\end{tabular}

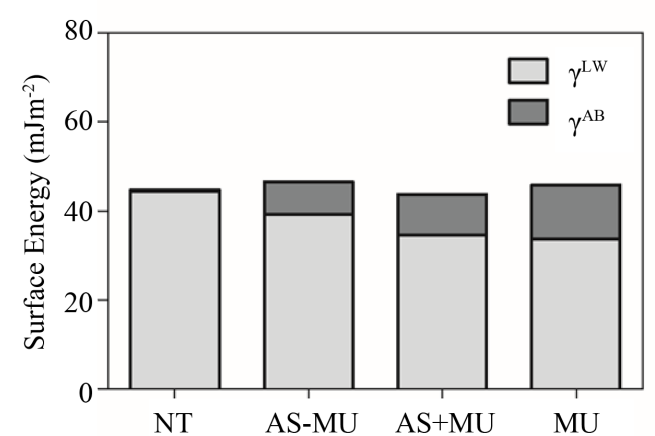

(a)

\begin{tabular}{ccccc}
\hline & NT & AS-Mu & AS+Mu & Mu \\
\hline $\boldsymbol{V}^{+}\left(\mathbf{m J m}^{-2}\right)$ & 0 & 0.2 & 0.28 & 0.70 \\
\hline$V^{-}\left(\mathbf{m J m}^{-2}\right)$ & 14.84 & 66.82 & 71.27 & 53.7 \\
\hline
\end{tabular}

(b)

\begin{tabular}{ccccc}
\hline & NT & AS-Mu & AS+Mu & Mu \\
\hline$\Delta \mathbf{G}_{\text {sws }}\left(\mathrm{mJm}^{-2}\right)$ & -40.1 & 47.3 & 55.4 & 33.2 \\
\hline
\end{tabular}

(c)

Figure 1. Free energy components and degree of hydrophobicity $\left(\Delta G_{s w s}\right)$ determined for the non-treated and treated polystyrene surfaces. The total surface tension, composed by the Lifshitz-van der Walls component $\gamma^{L W}$ and acid-base component $\gamma^{A B}$ (a), the electron-accepting $\gamma^{+}$and electron-donating $\gamma^{-}$parameters (b), as well as the degree of hydrophobicity $\left(\Delta G_{s w s}\right)(\mathrm{c})$ are shown. NT-non-treated polystyrene surface; AS - Mu-polystyrene surface treated with artificial saliva without mucin; AS + Mu-polystyrene surface treated with artificial saliva with mucin; Mu-polystyrene surface treated with mucin in PBS. 
Figure 1(a) shows a decrease on the Lifshitz-van der Walls component $\left(\gamma^{L W}\right)$ after treating the polystyrene surface with AS - Mu, AS + Mu and Mu. The presence of mucin on the conditioning medium (AS + Mu and Mu) induced a higher reduction on the $\gamma^{L W}$ component. Oppositely, treatments with $\mathrm{AS}-\mathrm{Mu}, \mathrm{AS}+\mathrm{Mu}$ and $\mathrm{Mu}$ increased the acid-base $\left(\gamma^{A B}\right)$ parameter of the free energy. Additionally, the electron-donating $\left(\gamma^{-}\right)$and electron-accepting $\left(\gamma^{+}\right)$parameter values increased significantly in the presence of AS or mucin (Figure 1(b)). For all the conditions, the $\gamma^{+}$component was smaller than the $\gamma^{-}$component (inferior to $1 \mathrm{mJm}^{-2}$ ).

Combining the contact angles and surface free energies (Table 1 and Figure 1), the hydrophobicity of the surfaces was determined as an expression of the free energy ( $\left.\Delta G_{s w s}\right)$ interacting between two identical surfaces (s) immersed in water (w) (Figure 1(c)).

The $\Delta G_{\text {sws }}$ of the polystyrene surface was found to be $-40.1 \mathrm{mJm}^{-2}$ (Figure $1(\mathrm{c})$ ), reflecting its hydrophobic character, since a hydrophobic surface is a surface for which $\Delta G_{s w s}<0$ [17]. Hydrophobic surfaces are usually surfaces with a low $\gamma^{-}$as well as a low $\gamma^{+}$parameter, which was observed for polystyrene (Figure 1(b)). The treatments with AS - Mu, AS + Mu and Mu significantly increased the $\Delta G_{s w s}$ values, changing the surface characteristic from hydrophobic to hydrophilic.

\subsection{Effect of the Growing Media on C. albicans and C. parapsilosis Surface Characteristics}

Interestingly, the four oral clinical isolates have different contact angle values, indicating different surface characteristics (Table 2). In fact, in the absence of mucin, C. parapsilosis AD had the highest water contact angle (> $50^{\circ}$ ) and C. albicans AM had the lowest water contact angle (approximately $30^{\circ}$ ).

Moreover, the presence of mucin in the medium strongly influenced the water contact angles of all Candida spp. a decrease on the water contact angle value was observed for C. albicans AC and C. parapsilosis AM2, while the opposite effect occurred for C. albicans AM and C. parapsilosis AD. All the formamide contact angles increased, except for $C$. parapsilosis AD. The $\alpha$-bromonaftalene contact angles also increased in the presence of mucin, except for C. parapsilosis AM2.

On the absence of mucin, C. albicans strains and C. parapsilosis AM2 had a $\gamma^{A B}$ between 9 and $13 \mathrm{mJm}^{-2}$, while C. parapsilosis AD presented a very small $\gamma^{A B}$ (around $2 \mathrm{mJm}^{-2}$ ) (Figure 2). When mucin was present in the medium, the $\gamma^{A B}$ of C. albicans AM and C. parapsilosis AM2 was near $0 \mathrm{mJm}^{-2}$, the $\gamma^{A B}$ of C. albicans AC decreased and $\gamma^{A B}$ of $C$. parapsilosis AD increased. The $\gamma^{L W}$ was stable when the medium was supplemented with mucin, which combined with $\gamma^{A B}$, resulted on a decrease of surface free energy for C. albicans AC, C. albicans AM and C. parapsilosis AM2, and in an increase for C. parapsilosis AD.

The electron-donating $\left(\gamma^{-}\right)$and electron-accepting $\left(\gamma^{+}\right)$parameters calculated for the Candida spp. are shown in Table 3(a). In the absence or presence of mucin, all the Candida spp. have a $\gamma^{+}$component smaller than the $\gamma^{-}$ component. C. albicans AM presented the highest $\left(47.6 \mathrm{mJm}^{-2}\right)$ and C. albicans AC the lowest $\left(21.33 \mathrm{mJm}^{-2}\right) \gamma^{-}$ values when compared to the other microorganisms. C. albicans AC had the higher $\gamma^{+}$value $\left(1.93 \mathrm{mJm}^{-2}\right)$ of all the Candida spp. When mucin is present, the surface characteristics of all Candida spp. are altered, with an increase in the $\gamma^{-}$parameter and a decrease on the $\gamma^{+}$value, except C. parapsilosis AD.

Table 2. Averaged contact angle values measured on Candida albicans AC, Candida albicans AM, Candida parapsilosis $\mathrm{AD}$ and Candida parapsilosis AM2, grown in artificial saliva without mucin or artificial saliva with mucin. Three standard liquids were used: water, formamide, and $\alpha$-bromonaftalene. Average \pm standard error (SE) are represented.

\begin{tabular}{|c|c|c|c|c|c|}
\hline & Cells & Strains & $\theta_{w}\left({ }^{\circ}\right) \pm S E$ & $\theta_{\mathrm{f}}\left({ }^{\circ}\right) \pm \mathrm{SE}$ & $\theta_{\mathrm{b}}\left({ }^{\circ}\right) \pm \mathrm{SE}$ \\
\hline \multirow{4}{*}{ AS without mucin } & \multirow{2}{*}{ C. albicans } & $\mathrm{AC}$ & $48.03 \pm 2.38$ & $19.20 \pm 1.98$ & $17.24 \pm 1.21$ \\
\hline & & AM & $29.28 \pm 1.97$ & $27.75 \pm 1.97$ & $22.02 \pm 2.02$ \\
\hline & \multirow{2}{*}{ C. parapsilosis } & $\mathrm{AD}$ & $54.47 \pm 215$ & $47.16 \pm 2.49$ & $26.41 \pm 2.02$ \\
\hline & & AM2 & $44.18 \pm 2.82$ & $28.04 \pm 1.41$ & $26.97 \pm 3.24$ \\
\hline \multirow{4}{*}{ AS with mucin } & \multirow{2}{*}{ C. albicans } & $\mathrm{AC}$ & $26.08 \pm 1.80$ & $39.33 \pm 3.23$ & $32.47 \pm 1.54$ \\
\hline & & $\mathrm{AM}$ & $38.14 \pm 1.02$ & $43.55 \pm 2.48$ & $23.34 \pm 1.34$ \\
\hline & \multirow{2}{*}{ C. parapsilosis } & $\mathrm{AD}$ & $57.62 \pm 1.55$ & $35.75 \pm 1.44$ & $33.59 \pm 1.86$ \\
\hline & & AM2 & $28.97 \pm 2.05$ & $54.06 \pm 3.54$ & $23.34 \pm 1.34$ \\
\hline
\end{tabular}


Table 3. Free energy components and degree of hydrophobicity $\left(\Delta G_{s w s}\right)$ determined for Candida spp. Electron-accepting $\gamma^{+}$ and electron-donating $\gamma^{-}$parameters of the acid-base component $\gamma^{A B}$ (a) and degree of hydrophobicity $\left(\Delta G_{s w s}\right)(b)$ determined for the four Candida spp. in artificial saliva without mucin and artificial saliva with mucin.

(a)

\begin{tabular}{cccccc}
\hline & & C. albicans AC & C. albicans AM & C. parapsilosis AD & C. parapsilosis AM2 \\
\hline \multirow{2}{*}{ AS without mucin } & $\gamma^{-}$ & 21.32 & 47.60 & 28.86 & 29.50 \\
& $\gamma^{+}$ & 1.93 & 0.44 & 0.03 & 1.30 \\
\multirow{2}{*}{ AS with mucin } & $\gamma^{-}$ & 60.79 & 49.78 & 17.00 & 76.20 \\
& $\gamma^{+}$ & 0.04 & 0.00 & 1.69 & 0.00 \\
\hline
\end{tabular}

(b

\begin{tabular}{cccccc} 
& & C. albicans AC & C. albicans AM & C. parapsilosis AD & C. parapsilosis AM2 \\
\hline AS without mucin & $\boldsymbol{\Delta} \mathbf{G}_{\text {sws }}$ & -19.9 & 20.2 & -4.6 & -4.7 \\
AS with mucin & $\boldsymbol{\Delta} \mathbf{G}_{\text {sws }}$ & 44.6 & 28.8 & -22.2 & 62.5 \\
\hline
\end{tabular}

AS without mucin

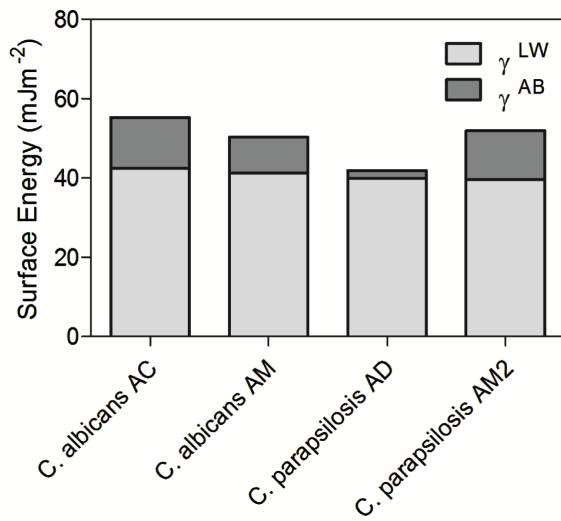

AS with mucin

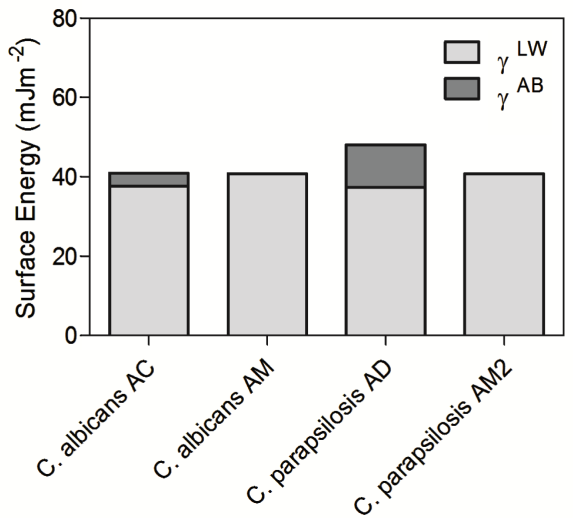

Figure 2. Free energy components determined for the Candida spp. in artificial saliva without mucin (a) and artificial saliva with mucin (b). The total surface tension, Lifshitz-van der Walls component $\gamma^{L W}$, acid-base component $\gamma^{A B}$, are shown.

On the absence of mucin, C. albicans AC and C. parapsilosis strains were found to be hydrophobic $\left(\Delta G_{s w s}<\right.$ 0 ) (Table 3(b)), while C. albicans AM was found to be hydrophilic $\left(\Delta G_{s w s}>0\right)$. Hydrophobic surfaces are usually surfaces with a low $\gamma^{-}$as well as a low $\gamma^{+}$parameter, which is in accordance with these results. The presence of mucin changed the surface characteristic of C. albicans AC and C. parapsilosis AM2 from hydrophobic to hydrophilic. The $\Delta G_{s w s}$ calculated for $C$. parapsilosis AD become even more negative, while for $C$. albicans AM it remained similar.

\subsection{Effect of Saliva and Mucin on the Adhesion of C. albicans and C. parapsilosis}

When mucin is not present in the growth medium, the adhesion of all Candida spp. is reduced only on the polystyrene surface treated with mucin $(\mathrm{Mu})$. The number of viable of $C$. parapsilosis AD cells increased when attached to the surface with AS-Mu treatment (Figure 3).

The two C. parapsilosis strains adhered at a higher extend to AS - Mu polystyrene than the two C. albicans strains. For the treatment of the polystyrene surface with $\mathrm{Mu}$ the adhesion followed the order: C. albicans AM > C. albicans $\mathrm{AC}>C$. parapsilosis $\mathrm{AD}=$ C. parapsilosis AM2.

It is also important to observe that the number of $C$. albicans AC cells adhered to the polystyrene surfaces increased when mucin was added. The same adhesion trend was observed for C. albicans AM, although there were no significant differences between the treatments with AS - Mu and AS + Mu. For the two C. parapsilosis 


\section{C. albicans AC}

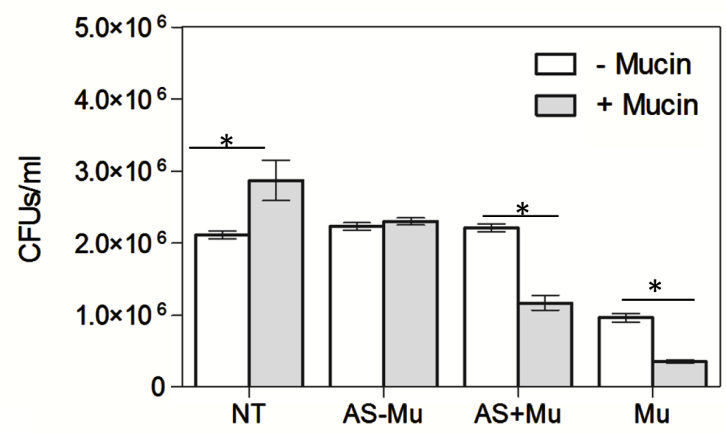

C. albicans AM

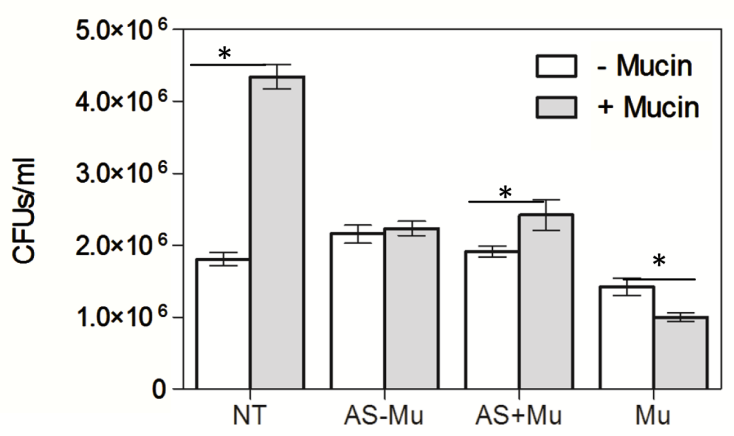

C. parapsilosis AD

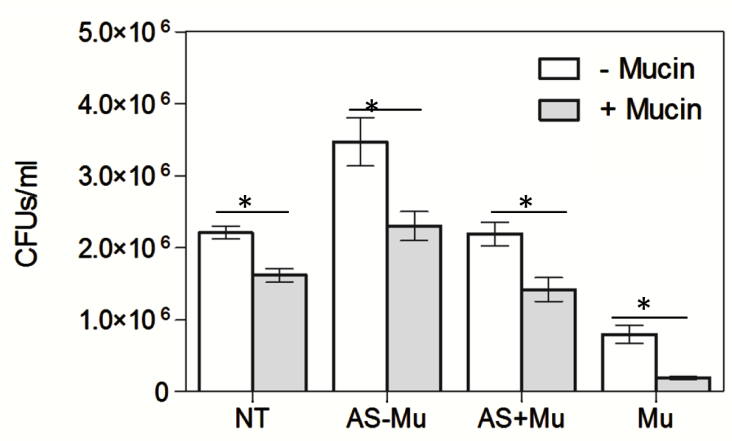

C. parapsilosis AM2

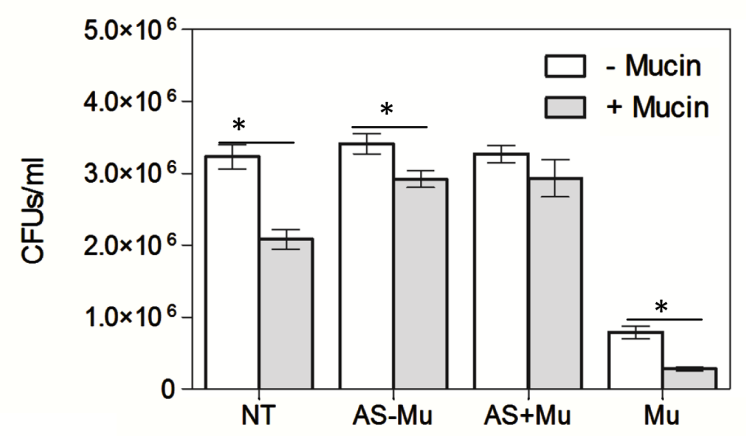

Figure 3. Number of oral clinical isolates of Candida spp. Adhered onto non-treated (NT) polystyrene or treated with artificial saliva without mucin (AS - Mu), artificial saliva with mucin (AS + Mu) and mucin in PBS (Mu).

strains only the treatment with Mu was able to significantly reduce the adhesion. Actually, the adhesion of $C$. parapsilosis $\mathrm{AD}$ and C. parapsilosis AM2 was increased for AS - Mu and AS - Mu/AS + Mu, respectively.

In the presence of mucin, the adhesion of $C$. albicans strains to the non-treated polystyrene surface was increased; while to the polystyrene treated with Mu was reduced. The adhesion to AS - Mu was not affected. The presence of mucin in the growth medium also reduced the adhesion of $C$. parapsilosis $\mathrm{AD}$ to all the surfaces, while the adhesion of C. parapsilosis AM2 was only reduced for the NT and Mu polystyrene surfaces.

\subsection{Energies of Adhesion of Candida spp. to the Polystyrene Surfaces}

In the absence and in the presence of mucin on the growth medium, the interaction energies between all the Candida spp. strains and the polystyrene surfaces treated with AS - Mu, AS + Mu and Mu were always unfavorable $\left(\Delta G_{a d h}>0\right)$ (Table 4). $\Delta G_{a d h}$ was higher for the AS + Mu, followed by AS - Mu and finally Mu. For the non-treated polystyrene surface, when grown in the absence of mucin, only C. albicans AM had an unfavorable $\Delta G_{a d h}$. When Candida spp. were in the presence of mucin, had $\Delta G_{a d h}>0$, except C. parapsilosis AD.

\section{Discussion}

The poor growth conditions on the oral environment oblige microbial cells to adhere in order to survive and colonize the oral cavity [7] [18]. Adhesion is a complex process, where surface free energies, van der Waals and electrostatic forces, hydrophobic interactions, cation bridging, receptor ligand binding, and the presence of nutrients are determinant for the adhesion of a microorganism [6]. In the particular case of the oral cavity colonization, the presence of saliva strongly influences adhesion. Saliva is a complex fluid secreted by the salivary glands, which forms a protective mechanical barrier against microbial colonization. It has antimicrobial properties given by different components such as lactoferrin, immunoglobulins, histatin or lysozymes [5]. Nevertheless, saliva is composed by other molecules, such as mucins and proline-rich-proteins that have been reported to 
Table 4. Interfacial free energies of adhesion between Candida spp. and polystyrene surfaces non-treated and treated with $\mathrm{AS}-\mathrm{Mu}, \mathrm{AS}+\mathrm{Mu}$, and $\mathrm{Mu}$. The total free energy $\left(\Delta G_{a d h}\right)$ is presented.

\begin{tabular}{|c|c|c|c|c|c|}
\hline & & C. albicans AC & C. albicans AM & C. parapsilosis AD & C. parapsilosis AM2 \\
\hline \multirow{4}{*}{$\begin{array}{l}\text { AS without } \\
\text { mucin }\end{array}$} & NT & -20.5 & 1.2 & -15 & -12 \\
\hline & AS - Mu & 13 & 38.8 & 28.2 & 22.7 \\
\hline & $\mathbf{A S}+\mathbf{M u}$ & 16.4 & 42.2 & 32 & 26 \\
\hline & Mu & 8.8 & 31.6 & 21.2 & 17.3 \\
\hline \multirow{4}{*}{$\begin{array}{l}\text { AS with } \\
\text { mucin }\end{array}$} & NT & 10.3 & 1.3 & -24.1 & 18.2 \\
\hline & AS - Mu & 50.9 & 44.6 & 10.3 & 59.9 \\
\hline & $\mathbf{A S}+\mathbf{M u}$ & 54.2 & 48.2 & 13.6 & 63.4 \\
\hline & Mu & 41.9 & 36 & 6 & 50.1 \\
\hline
\end{tabular}

facilitate adherence to surfaces [5]. There are contradicting reports on the literature regarding the influence of saliva on Candida adherence, with studies showing that the presence of saliva reduces the adherence of C. albicansin acrylic resin based materials while others demonstrate an increased adherence [19].

This study gives a new insight on the effect of saliva on Candida spp. adhesion, and the precise role of a single component-mucin. The effect of mucin on the microorganism surface characteristics as well as its influence on the surface properties of polystyrene was assessed.

As expected, a high contact angle value $\left(\sim 67^{\circ}\right)$ and a low free energy $\left(<10^{\circ}\right)$ were obtained for the non-treated polystyrene surface, demonstrating its hydrophobic nature. All the treatments performed (AS - Mu, AS + Mu, $\mathrm{Mu}$ ) changed the nature of the polystyrene surface from hydrophobic to hydrophilic (Figure 1 and Table 1). Nevertheless, there were no statistically significant differences in the total surface free energies calculated before and after treatments. Still, the treatments with AS - Mu, AS + Mu and Mu increased the $\gamma^{A B}$ free energy parameter, which means a higher hydration of the surface. The increase in the $\gamma^{-}$values observed on Figure 1(b) is another indication of decreased hydrophobicity while the increase on $\gamma^{+}$indicates an increase of the electron acceptor sites at the surface [17]. Even so, for all the conditions tested, the $\gamma^{+}$component was always smaller than the $\gamma^{-}$component, indicating that the surfaces are electron donating in nature. The results show that the presence of the artificial saliva components mask the hydrophobic nature of the polystyrene surface, due to the adsorption of ions such as sodium, calcium, potassium, present in the artificial saliva that will influence the interactions between the electron-acceptor and electron-donor groups [20] [21]. The changes observed in the presence of mucin (in PBS) may be explained by the conformational adaptation and exposure of its hydrophilic terminals [14] [22] when bound to the surface. It is known that, although most proteins adsorb preferentially onto lowenergy surfaces, some proteins, such as albumin, preferentially adsorb on substrates with surface energies that have a high polar (electron donor parameter $(\gamma)$ ) components [23]. This is a very important result, as it demonstrates the impact on the surface free energy parameters and on the hydrophobicity of the surface (Figure 1(c)) materials in the presence of different substances. The change on the physical-chemical characteristic influences the formation of the acquired enamel pellicle onto the oral surfaces and, therefore, microbial adhesion.

The contact angles measured on the yeast lawns showed important variations in the surface characteristics of the oral clinical isolates (Figure 2 and Table 2). In the absence of mucin, C. albicans AC and C. parapsilosis strains were found to be hydrophobic, while C. albicans AM was found to be hydrophilic (Table 3(b)). Also, both in the absence or the presence of mucin, all the Candida spp. presented a $\gamma^{+}$component smaller than the $\gamma^{-}$ component, indicating that the microorganisms are electron donners in nature (Table 3(a)).

When Candida spp. were grown in artificial saliva with mucin, C. albicans AC and C. parapsilosis AM2 changed from hydrophobic to hydrophilic (Table 3(a)). The presence of free mucin molecules in the medium, with the ability to bind to Candida cells surface [24] [25], lead to an adaptation of the Candida spp. Physicchemical characteristics.

The energies of adhesion calculated based on the surface free energies of the Candida spp. and the polystyrene surfaces could not explain the adhesion profile on its own. Unfavorable energies of adhesion between the cells and the surfaces (Table 4) did not result in decreased adhesions (Figure 3). When Candida spp. strains 
were cultured in artificial saliva without mucin, a similar adhesion profile was observed onto all surfaces except for the treatment with mucin in PBS $(\mathrm{Mu})$. This result points for the fact the presence of mucin, by itself, on the polystyrene surface, reduces adsorption, while the presence of artificial saliva on the polystyrene surfaces also has a role on the adhesion of Candida to oral surfaces, neutralizing the effect of mucin. In this sense, the adhesion of Candida cells was not only influenced by the adsorption of mucin to the surface, but also by the ionic strength and other components from the artificial saliva present in the polystyrene surface, which masks the inhibitory effect of mucin.

In the presence of artificial saliva with mucin, an increased number of C. albicans adhered to the non-treated polystyrene surface was observed, while for $C$. parapsilosis strains the adhesion was reduced (Figure 3 ). This suggests that mucin or/and ions, present in the artificial saliva medium, interact with the cells' surface favoring C. albicans adhesion by increasing the interactions of electron-acceptor and electron-donor between polystyrene and the cell surface [20]. For C. parapsilosis strains, the effect of the presence of mucin in the medium seems to result in cells less apt to adhere. The effect of the mucin on the polystyrene surface (treatment $\mathrm{Mu}$ ) further reduced adhesion of all the Candida strains while the treatments with As - Mu and AS + Mu were not always efficient on the reduction of adhesion (Figure 3). Interestingly, the presence of mucin in the fluid phase has a higher influence on the adhesion of Candida cells than when mucin is adsorbed onto the surface.

As mentioned before, adhesion is a very complex process, mediated by several factors such as surface free energies and hydrophobic interactions, but the interaction between adhesins and surface binding sites play a determinant role on the adhesion process of yeast [26]. Candida adhesion to oral surfaces is also mediated by cells surface receptors, mainly agglutinin-like sequence (ALS) that is hydrophobic and bound preferentially to the abiotic surfaces [27]. In fact, it is well known that C. parapsilosis has five ALS genes and six genes predicted for glycophosphatidylinositol-anchored protein 30 (Pga 30), but little is known about their role in adhesion [3] [28]. C. albicans has nine ALS genes involved on the adhesion mechanism [29]. The differences in C. parapsilosis and C. albicans ALS will probably explain the differences found on the adhesion of the both types of Candida strains.

\section{Conclusions}

This study shows that the role of mucin on Candida spp. adhesion is complex and must be carefully examined. The four Candida strains used in this study behave differently in the presence of mucin, showing either increased or decreased adhesion depending on the presence of mucin on the growing medium or on the polystyrene surface. Actually, while the presence of adhered mucin onto the surface decreases the adhesion of all the strains, the combination of mucin with artificial saliva diminishes this effect.

Although there is not a direct correlation between adhesion and the surface free energies of adhesion of these particular Candida strains, the presence of artificial saliva affects the physicochemical characteristics of the adherent surface, as well the hydrophobicity behaviour of the strains.

This study clearly demonstrates that it is important to evaluate the surface characteristics, as they will enhance or decrease the microbial attachment.

\section{Acknowledgements}

The authors thank the Project "BioHealth-Biotechnology and Bioengineering approaches to improve health quality”, Ref. NORTE-07-0124-FEDER-000027, co-funded by the Programa Operacional Regional do Norte (ON.2-O Novo Norte), QREN, FEDER. The would also like to thank the Fundação para a Ciência e Tecnologia for the Strategic Project Pest-OE/EQB/LA0023/2013 and Fundação para a Ciência e Tecnologia (FCT) for Ana Oliveira PhD Grant (SFRH/BD/68588/2010) and Catarina L. Seabra PhD Grant (SFRH/BD/89001/2012). The authors would also like to acknowledge Professora Rosário Oliveira, which is no longer with us, for her exceptional contribution and dedication to this work.

\section{References}

[1] Kabir, M.A. and Ahmad, Z. (2013) Candida Infections and Their Prevention. ISRN Preventive Medicine, $2013,13$. http://dx.doi.org/10.5402/2013/763628

[2] Kojic, E.M. and Darouiche, R.O. (2004) Candida Infections of Medical Devices. Clinical Microbiology Reviews, 17, 
255-267. http://dx.doi.org/10.1128/CMR.17.2.255-267.2004

[3] Silva, S., Negri, M., Henriques, M., Oliveira, R., Williams, D.W. and Azeredo, J. (2011) Adherence and Biofilm Formation of Non-Candida albicans Candida Species. Trends Microbiology, 19, 241-247. http://dx.doi.org/10.1016/j.tim.2011.02.003

[4] Coronado-Castellote, L. and Jiménez-Soriano, Y. (2013) Clinical and Microbiological Diagnosis of Oral Candidiasis, Journal of Clinical and Experimental Dentistry, 5, e279-e286. http://dx.doi.org/10.4317/jced.51242

[5] Dodds, M.W., Johnson, D.A. and Yeh, C.K. (2005) Health Benefits of Saliva: A Review. Journal of Dentistry, 33, 223-233. http://dx.doi.org/10.1016/j.jdent.2004.10.009

[6] Linden, S.K., Sutton, P., Karlsson, N.G., Korolik, V. and McGuckin, M.A. (2008) Mucins in the Mucosal Barrier to Infection. Mucosal Immunology, 1, 183-197. http://dx.doi.org/10.1038/mi.2008.5

[7] Cannon, R.D. and Chaffin, W.L. (1999) Oral Colonization by Candida albicans. Critical Reviews in Oral Biology \& Medicine, 10, 359-383. http://dx.doi.org/10.1177/10454411990100030701

[8] Cannon, R.D., Holmes, A.R., Mason,A.B. and Monk, B.C. (1995) Oral Candida: Clearance, Colonization, or Candidiasis? Journal of Dental Research, 74, 1152-1161. http://dx.doi.org/10.1177/00220345950740050301

[9] Elguezabal, N., Maza, J.L., Dorronsoro, S. and Ponton, J. (2008) Whole Saliva Has a Dual Role on the Adherence of Candida albicans to Polymethylmetacrylate, The Open Dentistry Journal, 2, 1-4.

[10] Vasilas, A., Molina, L., Hoffman, M. and Haidaris, C.G. (1992) The Influence of Morphological Variation on Candida albicans Adhesion to Denture Acrylic in Vitro. Archives of Oral Biology, 37, 613-622. http://dx.doi.org/10.1016/0003-9969(92)90123-P

[11] Edgerton, M., Scannapieco, F.A., Reddy, M.S. and Levine, M.J. (1993) Human Submandibular-Sublingual Saliva Promotes Adhesion of Candida albicans to Polymethylmethacrylate. Infect Immunology, 61, 2644-2652.

[12] Lamfon, H., Porter, S.R., McCullough, M. and Pratten, J. (2003) Formation of Candida albicans Biofilms on NonShedding Oral Surfaces. European Journal of Oral Sciences, 111, 465-471. http://dx.doi.org/10.1111/j.0909-8836.2003.00084.x

[13] Guggenheim, B., Giertsen, E., Schupbach, P. and Shapiro, S. (2001) Validation of an in Vitro Biofilm Model of Supragingival Plaque. Journal of Dental Research, 80, 363-370. http://dx.doi.org/10.1177/00220345010800011201

[14] Busscher, H.J., Bos, R. and Van der Mei, H.C. (1995) Initial Microbial Adhesion Is a Determinant for the Strength of Biofilm Adhesion. FEMS Microbiology Letters, 128, 229-234. http://dx.doi.org/10.1111/j.1574-6968.1995.tb07529.x

[15] Van Oss, C.J., Chaudhury, M.K. and Good, R.J. (1987) Monopolar Surfaces. Advances in Colloid Interface Science, 28, 35-64. http://dx.doi.org/10.1016/0001-8686(87)80008-8

[16] Van Oss, C.J., Ju, L., Chaudhury, M.K. and Good, R.J. (1989) Estimation of the Polar Parameters of the Surface Tension of Liquids by Contact Angle Measurements on Gels. Journal of Colloid and Interface Science, 128, 313-319. http://dx.doi.org/10.1016/0021-9797(89)90345-7

[17] Van Oss, C.J. (1995) Hydrophobic, Hydrophilic and Other Interactions in Epitope-Paratope Binding. Molecular Immunology, 32, 199-211. http://dx.doi.org/10.1016/0161-5890(94)00124-J

[18] Henriques, M., Gasparetto, K., Azeredo, J. and Oliveira, R. (2002) Experimental Methodology to Quantify Candida albicans Cell Surface Hydrophobicity. Biotechnology Letters, 24, 1111-1115. http://dx.doi.org/10.1023/A:1016083021525

[19] Pereira-Cenci, T., Del Bel Cury, A.A., Crielaard, W. and Ten Cate, J.M. (2008) Development of Candida-Associated Denture Stomatitis: New Insights. Journal of Applied Oral Science, 16, 86-94. http://dx.doi.org/10.1590/S1678-77572008000200002

[20] Henriques, M., Azeredo, J. and Oliveira, R. (2004) Adhesion of Candida albicans and Candida dubliniensis to Acrylic and Hydroxyapatite. Colloids and Surfaces B: Biointerfaces, 33, 235-241. http://dx.doi.org/10.1016/j.colsurfb.2003.10.012

[21] Oliveira, R. (1997) Understanding Adhesion: A Means for Preventing Fouling. Experimental Thermal and Fluid Science, 14, 316-322. http://dx.doi.org/10.1016/S0894-1777(96)00134-3

[22] Busscher, H.J., Geertsema-Doornbusch, G.I. and Van der Mei, H.C. (1997) Adhesion to Silicone Rubber of Yeasts and Bacteria Isolated from Voice Prostheses: Influence of Salivary Conditioning Films. Journal of Biomedical Materials Research, 34, 201-209. http://dx.doi.org/10.1002/(SICI)1097-4636(199702)34:2<201::AID-JBM9>3.0.CO;2-U

[23] Comelles, J., Estévez, M., Martínez, E. and Samitier, J. (2010) The Role of Surface Energy of Technical Polymers in Serum Protein Adsorption and MG-63 Cells Adhesion. Nanomedicine: Nanotechnology, Biology and Medicine, 6, 4451. http://dx.doi.org/10.1016/j.nano.2009.05.006

[24] Hoffman, M.P. and Haidaris, C.G. (1993) Analysis of Candida albicans Adhesion to Salivary Mucin. Infection and Immunity, 61, 1940-1949. 
[25] Schenkels, L.C., Veerman, E.C. and Nieuw-Amerongen, A.V. (1995) Biochemical Composition of Human Saliva in Relation to Other Mucosal Fluids. Critical Reviews in Oral Biology \& Medicine, 6, 161-175. http://dx.doi.org/10.1177/10454411950060020501

[26] Desai, C., Mavrianos, J. and Chauhan, N. (2011) Candida glabrata Pwp7p and Aed1p Are Required for Adherence to Human Endothelial Cells. FEMS Yeast Research, 11, 595-601. http://dx.doi.org/10.1111/j.1567-1364.2011.00743.x

[27] Aoki, W., Kitahara, N., Miura, N., Morisaka, H., Kuroda, K. and Ueda, M. (2012) Profiling of Adhesive Properties of the Agglutinin-Like Sequence (ALS) Protein Family, a Virulent Attribute of Candida albicans. FEMS Immunology \& Medical Microbiology, 65, 121-124. http://dx.doi.org/10.1111/j.1574-695X.2012.00941.X

[28] Butler, G., Rasmussen, M.D., Lin, M.F., Santos, M.A., Sakthikumar, S., Munro, C.A., Rheinbay, E., Grabherr, M., Forche, A., Reedy, J.L., Agrafioti, I., Arnaud, M.B., Bates, S., Brown, A.J., Brunke, S., Costanzo, M.C., Fitzpatrick, D.A., Groot, P.W., Harris, D., Hoyer, L.L., Hube, B., Klis, F.M., Kodira, C., Lennard, N., Logue, M.E., Martin, R., Neiman, A.M., Nikolaou, E., Quail, M.A., Quinn, J.M., Santos, C., Schmitzberger, F.F., Sherlock, G., Shah, P., Silverstein, K.A., Skrzypek, M.S., Soll, D., Staggs, R., Stansfield, I., Stumpf, M.P., Sudbery, P.E., Srikantha, T., Zeng, Q., Berman, J., Berriman, M., Heitman, J., Gow, N.A., Lorenz, M.C., Birren, B.W., Kellis, M. and Cuomo, C.A. (2009) Evolution of Pathogenicity and Sexual Reproduction in Eight Candida Genomes. Nature, 459, 657-662. http://dx.doi.org/10.1038/nature08064

[29] Hoyer, L.L. (2001) The ALS Gene Family of Candida albicans. Trends in Microbiology, 9, 176-180. http://dx.doi.org/10.1016/S0966-842X(01)01984-9 